\title{
Article \\ A Fuzzy Method for Joint Resource Allocation and Stable Pairing in D2D Communications
}

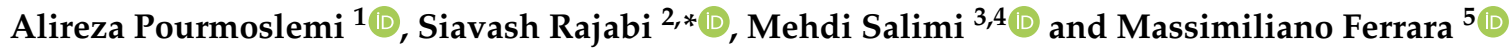 \\ 1 Department of Mathematics, Payame Noor University, Tehran P.O. Box 19395-4697, Iran; \\ a_pourmoslemy@pnu.ac.ir \\ 2 Department of Electrical Engineering, Hamedan University of Technology, Hamedan 65155, Iran \\ 3 Department of Mathematics \& Statistics, St. Francis Xavier University, Antigonish, NS B2G 2W5, Canada; \\ msalimi@stfx.ca \\ 4 Center for Dynamics, Faculty of Mathematics, Technische Universität Dresden, 01062 Dresden, Germany \\ 5 DiGiES \& Decisions Lab, Università Mediterranea di Reggio Calabria, 89124 Reggio Calabria, Italy; \\ massimiliano.ferrara@unirc.it \\ * Correspondence: siavash.rajabi@hut.ac.ir
}

check for updates

Citation: Pourmoslemi, A.; Rajabi, S.; Salimi, M.; Ferrara, M. A Fuzzy Method for Joint Resource Allocation and Stable Pairing in D2D

Communications. Appl. Sci. 2022, 12, 1343. https://doi.org/10.3390/ app12031343

Academic Editor: Amalia Miliou

Received: 14 November 2021

Accepted: 21 January 2022

Published: 27 January 2022

Publisher's Note: MDPI stays neutral with regard to jurisdictional claims in published maps and institutional affiliations.

Copyright: () 2022 by the authors Licensee MDPI, Basel, Switzerland. This article is an open access article distributed under the terms and conditions of the Creative Commons Attribution (CC BY) license (https:// creativecommons.org/licenses/by/ $4.0 /)$.

\begin{abstract}
In this paper, a device-to-device (D2D) communications multiple-metric scenario for resource allocation and pairing is studied. To improve the D2D connection stability, a fuzzy method based on the data rate and battery levels of potential D2D pairs is proposed in such a way that the set of D2D transmitters is considered as a fuzzy set. A single cell scenario with a cellular user and some co-channel D2D nodes consists of D2D receivers, and transmitters are taken into account. Furthermore, a stable fuzzy pairing criterion is proposed for the selection of the best D2D transmitter. The proposed method is compared with three other pairing methods named the maximum sum rate, constant pairing method, and random pairing method. The simulation results show that the mentioned pairing method outperforms the other three methods in terms of stability and fairness criteria and follows the maximum sum-rate method from the sum-rate criteria point of view.
\end{abstract}

Keywords: device to device communications; fuzzy pairing method; $t$-norms; einstein sum

\section{Introduction and Related Works}

The increase in the number of wireless networks users and applications has led to an increase in demand for the use of cellular network resources. This challenge has led to the formation of services that reduce the load on the central part of the network. D2D communications is a proximity-based service that enables two devices to exchange information without the need for a base station and standardized by the 3rd Generation Partnership Project (3GPP) Long-Term Evolution (LTE). D2D communications are divided into overlay in-band, underlay in-band, and out-band communications. In in-band communications, D2D users utilize the cellular network spectrum that is categorized to overlay and underlay [1].

The underlay in-band is a D2D communications mode that allows the D2D users to use the cellular spectrum and causes the interference to cellular links and vice versa. On the other hand, the cellular spectrum is distributed between cellular and D2D users in overlay in-band mode with no interference. In out-band communications, users use a different spectrum other than the cellular spectrum. The main advantages of D2D service are the optimal allocation of cellular network resources, power consumption, the cellular network traffic offloading, and the possibility of creating public safety networks [2].

\subsection{Pairing in D2D Communications}

In the history of D2D communications, it is often supposed that the D2D pairs are formed before. The main issues are selecting the proper cellular user to share resources 
with, selecting the operating mode, and setting the power for cellular and D2D users. In the literature, pairing is defined and investigated as permitting a D2D connection (a transmitter and its associated receiver) to use the resource of a cellular connection [3-6]. However, stable pairing for a D2D user in a practical scenario is an essential problem.

This problem is investigated and studied in various approaches in the literature [7-13]. The nearest transmitter is considered to be the best transmitter in [7-10], while the nearest transmitter is not necessarily the best [11-13]. Our approach to pairing is similar to [11-13], except that we consider the problem of multi-user pairing and resource allocation.

In [14], the interactions and correlations among user equipments (UEs) and an iterative power allocation algorithm with a game-theoretic approach is studied to analyze establish mutual preferences based on nonlinear fractional programing. Then, a Gale-Shapley algorithm is employed to match D2D pairs with cellular UEs.

A novel two-timescale resource allocation scheme is investigated in [15], so that the pairing between CUs and D2D pairs is decided at a long timescale. Moreover, the transmission time for the CU and D2D pair is presented at a short timescale. Specifically, the optimal cooperation policy to decide the transmission time based on the instantaneous channel state information (CSI) is investigated to characterize the long-term payoff of each potential CU-D2D pair. They prove that the optimal policy is a threshold policy that can be achieved via binary search.

In [16], the authors solve a combinatorial NP-hard problem. User equipments (UEs) from two finite and disjoint sets are matched in a two-sided stable way based on the mutual preferences in each stage. First, the preferences of UEs are defined as the maximum achievable EE. An iterative power allocation algorithm is proposed to optimize EE under a specific match, which is developed by exploiting nonlinear fractional programming and Lagrange dual decomposition. Second, the authors propose an iterative matching algorithm, which first produces a stable match based on the fixed preferences and then dynamically updates the preferences according to the latest matching results in each iteration.

In all the mentioned articles except [11-13], the nearest transmitter is considered to be the best transmitter, while the nearest transmitter is not necessarily the best due to interference and battery level. In other articles, it is assumed that the D2D users are paired and their proposed algorithms focused on resource allocation of D2D users with proper cellular users. Our proposed method shows that more suitable options can be found for pairing in D2D communications.

There are limited works in the literature consider the battery of UEs in D2D-based cellular communications [17-19]. Ref. [17] takes into account the remaining battery time of relay nodes to improve the performance of D2D communications. Ref. [18] proposes a spectrum sharing algorithm to maximize the energy efficiency of D2D communications that enhances the battery lifetime of UEs. Finally, Ref. [19] presented a relay discovery mechanism to optimize the periodic discovery transmission of D2D UEs.

\subsection{Fuzzy Mathematics}

L. A Zadeh introduced fuzzy sets in his significant paper [20] in 1965. His idea was to introduce a generalization of the concept crisp sets. The idea was to use numbers in $[0,1]$ as the membership degrees of elements instead of 0 and 1.

Definition 1 ([21]). Suppose that $X$ is a collection of objects. Then, $A=\{(x, \mu(x)): x \in X\}$ is called a fuzzy subset of $X$, where $\mu: X \rightarrow[0,1]$ is a function called membership function which denotes the membership degrees of elements in $X$.

In 1966, an application of fuzzy sets to find a pattern classification was introduced by Bellman, Kalaba, and Zadeh [22]. Four years later, a decision-making problem was solved by Zadeh and Bellman [23]. In the 1970s and 1980s, decision making, control, and pattern classification received the most attention by researchers [24-26]. The fuzzy mathematics applications were expanded in the following years. Another important application of fuzzy 
sets appeared to use fuzzy controllers [27]. Fuzzy genetics algorithms and neuro-fuzzy systems were studied in the 1990s [28]. Pattern recognition, machine learning, and wireless sensor networks are other fields of study that benefit from fuzzy mathematics [28-30].

Moreover, the applications of fuzzy mathematics in D2D communications have mostly been in the field of resource allocation and power control. A continuous fuzzy power control scheme is studied in [31] in such a way that the cellular user can communicate in low power to achieve better D2D users connection quality. A resource allocation algorithm is modeled through fuzzy mathematics and game theory in [32] to introduce an uncertain relationship between the resource and user. In [33], a fuzzy clustering algorithm is proposed to divide D2D users into several groups to reuse the cellular user resources and repress the co-channel interference. In [34], a resource allocation based on fuzzy clustering is proposed to solve the spectrum scarcity problem. A fuzzy logic-based vehicle handover algorithm is studied in [35] to select a proper access network.

In this study, we consider each transmitter as a fuzzy node in a D2D mobile network in such a way that a set of transmitters together with a membership function is regarded as a fuzzy set. The membership function assigns to each transmitter a number in $[0,1]$ based on the transmitter's data rate and battery level. Then, a decision-making function chooses the best transmitter with the highest fuzzy degree.

We use triangular norms and co-norms to build our fuzzy membership function.

Definition 2 (Ref. [36]). Let $T:[0,1] \times[0,1] \rightarrow[0,1]$ be a commutative, associative, and non-decreasing function which $T(x, 1)=x$, for all $x \in[0,1]$. Then, $T$ is called a triangular norm (t-norm).

For example, basic $t$-norms that will be called Lukasiewicz, Minimum, and Product $t$-norms are defined as follows, respectively.

1. $T_{L}(x, y)=\max (x+y-1,0)$,

2. $T_{M}(x, y)=\min (x, y)$,

3. $T_{P}(x, y)=x \cdot y$.

Schweizer and Sklar introduced $t$-conorms as dual operations of $t$-norms in 1961 [37].

Definition 3 ([36]). Let $S:[0,1] \times[0,1] \rightarrow[0,1]$ be an associative, commutative, and monotone function that $S(x, 0)=x$ for all $x \in[0,1]$. Then, $S$ is called triangular conorm (t-conorm).

Corresponding to basic $t$-norms, there are some $t$-conorms written as follows.

1. $S_{L}(x, y)=\min (x+y, 1)$, (Lukasiewicz t-conorm);

2. $S_{M}(x, y)=\max (x, y)$, (Maximum $t$-conorm);

3. $S_{P}(x, y)=x+y-x \cdot y$, (Probabilistic sum).

Another $t$-conorm, named the Einstein sum, is used in this paper to build our membership function [36]. The Einstein sum $\tau:[0,1] \times[0,1] \rightarrow[0,1]$ is defined as follows for all $x, y \in[0,1]$.

$$
\tau(x, y)=\frac{x+y}{1+x y} .
$$

In order to find a proper membership function, the Einstein sum is chosen after examining several $t$-norms and $t$-conorms. Other suitable triangular norms and conorms can be examined for future research. For more details about applications of the Einestein sum in fuzzy systems, see [3,38,39].

\subsection{Contributions}

The main contributions of this paper are summarized as follows.

1. A joint resource allocation and pairing scenario in D2D communications is investigated. A number of D2D receivers are considered for the reuse of cellular network 
resources. For each D2D receiver, there exists a number of potential D2D transmitters, and a stable fuzzy pairing criteria is proposed for the selection of the best transmitter.

2. The set of transmitters is considered as a fuzzy set in such a way that a fuzzy degree is assigned to each node, concerning the data rates and battery levels in connection with their potential receiver. We claim that this fuzzy membership function can be considered as a measure of the stability of the connections.

3. To examine the proposed method, this method is compared with three other pairing methods, so that the three parameters of stability, fairness, and sum rate in different modes such as changing the D2D search radius and increasing the number of D2D transmitters and receivers for all four methods are investigated.

4. The proposed method for D2D pairing, in addition to reducing the traffic load of the cellular network, leads to more stable connections with a higher quality of service. One of the significant applications of this method is that bulky content can be transferred more reliably.

\section{System Model}

In this paper, we consider the problem of joint resource allocation and stable pairing in an underlay in-band D2D enabled cellular communication network. The system model consists of a single cell with a cellular user and a number of co-channel D2D nodes. Some D2D nodes are transmitters and some are receivers, in such a way that there are several potential transmitters for each receiver. The transmitters of each receiver are specified separately to share different contents (Figure 1). In this scenario, it is assumed that the potential D2D transmitters around a receiver have a similar content that may be different from the potential D2D transmitters of the other D2D receiver. Therefore, different D2D categories (that consist of a D2D receiver and a number of potential D2D transmitters) are assumed to have different contents. Moreover, the considered scenario is a centralized protocol in which all the decisions are made by the base station. So, the set of potential D2D transmitters for each receiver are specified by their contents based on the base station decision.

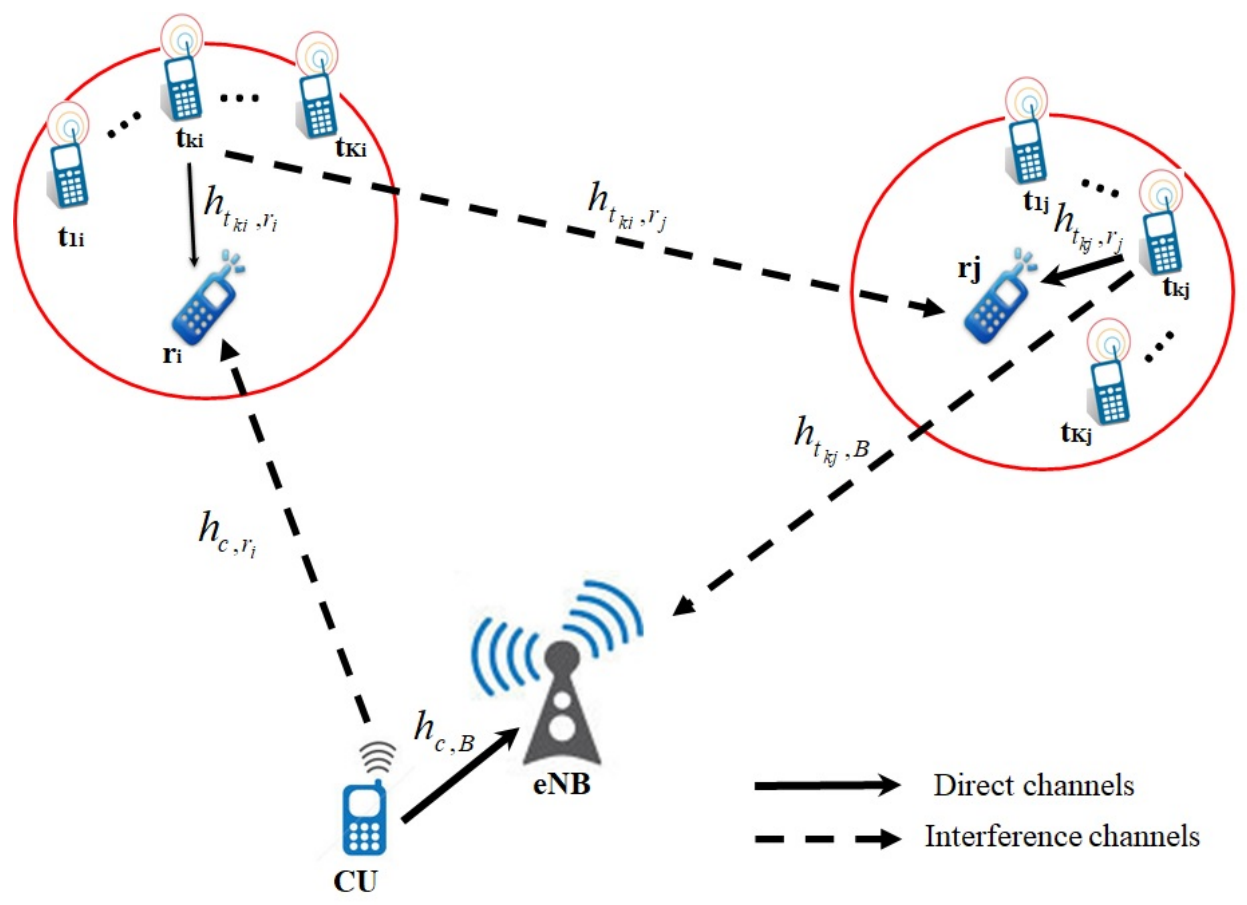

Figure 1. System model.

We tend to find the most suitable transmitter for pairing with each receiver so that stability is maintained. By stability, we mean a situation in which the battery levels and 
the data rates of two potential paired nodes are acceptable. For this purpose, considering potential transmitting D2D users as fuzzy nodes, the normalized smartphone battery levels and data rates are used to obtain an index for stability. Note that because of interference considerations, a D2D receiver may not be allowed to pair with any of the transmitters.

Let $\mathcal{D}_{r}=\left\{r_{1}, r_{2}, \ldots, r_{N}\right\}$ be the set of D2D receivers, where $N \in \mathbb{N}$ is the maximum number of co-channel D2D receivers with a cellular user in the network. For each D2D receiver $r_{i}(i=1, \ldots, N)$, the set $\mathcal{D}_{t_{i}}=\left\{t_{1 i}, t_{2 i}, \ldots, t_{K i}\right\}$ denotes the potential D2D transmitters, where $K \in \mathbb{N}$ is the number of D2D transmitters around the receiver $r_{i}$. Moreover, the D2D transmitters are located in a circle centered on each D2D receiver with radius $R_{\max }$, where $R_{\max }$ is the maximum search radius of D2D receivers.

The notations used in this paper are summarized in Table 1.

Table 1. Notation summary.

\begin{tabular}{|c|c|}
\hline Notation & Description \\
\hline$r_{i}$ & $i^{\text {th }} \mathrm{D} 2 \mathrm{D}$ receiver \\
\hline$t_{k i}$ & $k^{\text {th }}$ Potential D2D transmitter for $r_{i}$ D2D receiver \\
\hline$N$ & The maximum number of co-channel D2D receivers \\
\hline K & The number of D2D transmitters around each receiver \\
\hline$R_{\max }$ & The maximum search radius of D2D receivers \\
\hline$h_{c, B}$ & The direct channel between the cellular user and eNB \\
\hline$d_{c, B}$ & The distance between the cellular user and eNB \\
\hline$h_{t_{k i}, r_{j}}$ & The channel between the D2D transmitter $t_{k i}$ and the D2D receiver $r_{j}$ \\
\hline$d_{t_{k i}, r_{j}}$ & The distance between the D2D transmitter $t_{k i}$ and the D2D receiver $r_{j}$ \\
\hline$h_{t_{k i}, B}$ & The interference channel between the D2D transmitter $t_{k i}$ and eNB \\
\hline$d_{t_{k i}, B}$ & The distance between the D2D transmitter $t_{k i}$ and eNB \\
\hline$h_{c, r_{i}}$ & The interference channel between the cellular user and D2D receiver $r_{i}$ \\
\hline$d_{c, r_{i}}$ & The distance between the cellular user and D2D receiver $r_{i}$ \\
\hline $\mathrm{SINR}_{B}$ & The cellular link SINR \\
\hline$R_{B}$ & The cellular link data rate \\
\hline $\mathrm{SINR}_{k i}$ & The D2D link (consists of transmitter $t_{k i}$ and receiver $r_{i}$ ) SINR \\
\hline$R_{k i}$ & The D2D link (consists of transmitter $t_{k i}$ and receiver $r_{i}$ ) data rate \\
\hline$P^{c}$ & Cellular user's power \\
\hline$P^{d}$ & D2D transmitters' power \\
\hline$\alpha$ & Path loss exponent \\
\hline$N_{0}$ & The received noise power \\
\hline$E_{k i}$ & The battery level of transmitter $t_{k i}$ \\
\hline$E_{0}$ & The battery level threshold \\
\hline$R_{0}$ & The date-rate threshold \\
\hline
\end{tabular}

\subsection{Communication Models}

The system model channels are supposed to be independent and identically distributed (i.i.d.) Rayleigh channels with perfect channel state information (CSI) only available for the D2D receivers mentioned as follows:

1. $h_{c, B}$ and $d_{c, B}$ are the direct channel and distance between the cellular user and evolved node base station (eNB), respectively. 
2. $h_{t_{k i}, r_{j}}$ and $d_{t_{k i}, r_{j}}$ are the channel and distance between the D2D transmitter $t_{k i}$ and the D2D receiver $r_{j}$, respectively where $t_{k i} \in \mathcal{D}_{t_{i}}, r_{j} \in \mathcal{D}_{r}, k \in\{1, \ldots, K\}$ and $i, j \in\{1, \ldots, N\}$.

3. $h_{t_{k i}, B}$ and $d_{t_{k i}, B}$ are the interference channel and distance between the D2D transmitter $t_{k i}$ and eNB, respectively.

4. $\quad h_{\mathcal{C}, r_{i}}$ and $d_{\mathcal{C}, r_{i}}$ are the interference channel and distance between the cellular user and D2D receiver $r_{i}$, respectively.

The cellular and D2D links SINR can be noted as follows:

$$
\begin{aligned}
& \operatorname{SINR}_{B}=\frac{S_{B}}{I_{B}+N_{0}}, \\
& \operatorname{SINR}_{k i}=\frac{S_{k i}}{I_{i}+N_{0}},
\end{aligned}
$$

where

$$
\begin{gathered}
S_{B}=P^{c}\left|h_{c, B}\right|^{2}\left|d_{c, B}\right|^{-\alpha}, \\
S_{k i}=P^{d} \beta_{k i}\left|h_{t_{k i}, r_{i}}\right|^{2}\left|d_{t_{k i}, r_{i}}\right|^{-\alpha}, \\
I_{B}=\sum_{j=1}^{N} \sum_{k=1}^{K} \beta_{k j} P^{d}\left|h_{t_{k j}, B}\right|^{2}\left|d_{t_{k j}, B}\right|^{-\alpha}, \\
I_{i}=\sum_{j=1}^{N} \sum_{k^{\prime}=1}^{K} \beta_{k^{\prime} j} P^{d}\left|h_{t_{k^{\prime} j} r_{i}}\right|^{2}\left|d_{t^{\prime} j^{\prime}, r_{i}}\right|^{-\alpha}+P^{c}\left|h_{c, r_{i}}\right|^{2}\left|d_{c, r_{i}}\right|^{-\alpha} .
\end{gathered}
$$

Note that $N_{0}$ is the received noise power and $\beta_{k j}$ is the binary pairing variable denoted by

$$
\beta_{k j}= \begin{cases}1, & \text { if } t_{k j} \text { is paired with the receiver } r_{j} \\ 0, & \text { otherwise. }\end{cases}
$$

According to the above SINR denotations, the cellular and D2D users data rates are written as

$$
\begin{aligned}
& R_{B}=\log \left(1+\operatorname{SINR}_{B}\right) ; \\
& R_{k i}=\log \left(1+\operatorname{SINR}_{k i}\right) .
\end{aligned}
$$

\subsection{Construing the Fuzzy Membership Function}

In the mentioned network, a stable connection is a connection in which the data rate is acceptable while the link does not disconnect due to a low battery condition. The set of all nodes suitable for pairing has a fuzzy interpretation. Let the set $T=\left\{t_{k i} \mid t_{k i}\right.$ is a stable transmitter $\}$ be the fuzzy set, where $k \in\{1, \ldots, K\}$ and $i \in\{1, \ldots, N\}$. We tend to construct a fuzzy membership function for each node in $T$. Two network parameters, the normalized data rate $(R)$ and battery level $(E)$ of each node, are considered. To measure the stability, two thresholds $R_{0}$ and $E_{0}$ are set. Considering the limitations of the network protocols, these thresholds guarantee the stability of the D2D link.

Concerning the mentioned explanations, the proposed membership function $\mu: T \rightarrow[0,1]$ is defined as follows.

$$
\mu\left(t_{k i}\right)=\lambda\left(R_{k i}, E_{k i}\right)
$$


where $E_{k i}$ is the battery level of transmitter $t_{k i}$, and

$$
\lambda\left(R_{k i}, E_{k i}\right)= \begin{cases}R_{k i} ; & \left(E_{0} \leq E_{k i} \leq 1,\right. \\ E_{k i} ; & \left.0 \leq R_{k i}<R_{0}\right) \\ & \left(0 \leq E_{k i}<E_{0}\right. \\ \tau\left(R_{k i}, E_{k i}\right)\left(R_{k i}^{2}+E_{k i}^{2}\right) ; & \left(0<R_{k i} \leq 1\right) \\ & \left.0<R_{k i}<E_{0}\right) \\ \tau\left(R_{k i}, E_{k i}\right) ; & \left(E_{0} \leq E_{k i} \leq 1,\right. \\ & \left.R_{0} \leq R_{k i} \leq 1\right) .\end{cases}
$$

Note that the Einstein sum is applied as

$$
\tau\left(R_{k i}, E_{k i}\right)=\frac{R_{k i}+E_{k i}}{1+R_{k i} E_{k i}} .
$$

Since those fuzzy nodes with a high membership degree have both a higher battery level and a higher data rate among their neighbors, it can be said that the proposed membership function is an appropriate metric for measuring stability. For this reason, when transferring high volume contents, the connection is less likely to fail.

\section{Simulation Results}

In this section, four different criteria for pairing in a D2D communication scenario are investigated. These four methods are

- $\quad$ Fuzzy-based pairing (FP) method;

- Max-sum-rate pairing (MSP) method;

- $\quad$ Random pairing (RP) method;

- Constant pairing $(\mathrm{CP})$ method.

In the MSP method, the selection of the desired transmitter for D2D communication is based on the highest sum rate. According to (4) and (5), the sum rate of the co-channel cellular and potential D2D pairs are calculated as $R_{B}+\sum_{i=1}^{N} R_{k i}$. In the RP method, the D2D transmitters are selected randomly. Finally, the nearest transmitter is considered as the paired transmitter in the $\mathrm{CP}$ method. The main simulation parameters are summarized in Table 2. Note that the simulation parameters are according to [40].

Table 2. Simulation parameters.

\begin{tabular}{cc}
\hline Parameter & Value \\
\hline$R_{c}$ (Cellular radius) & $500 \mathrm{~m}$ \\
\hline$R_{\max }$ (D2D search radius) & $200 \mathrm{~m}$ \\
\hline $\mathrm{N}$ (The number of D2D receivers for each cellular users) & $1-4$ \\
\hline $\mathrm{K}$ (The number of D2D transmitters for each receiver) & $1-4$ \\
\hline$P^{c}$ (Cellular user's power) & $23 \mathrm{dBm}$ \\
\hline$P^{d}$ (D2D transmitters' power) & $15 \mathrm{dBm}$ \\
\hline$\alpha$ (Path loss exponent) & 3.5 \\
\hline$R_{0}$ (Data-rate threshold) & 0.3 \\
\hline$E_{0}$ (Battery-level threshold) & 0.25 \\
\hline
\end{tabular}

Now, we compare the pairing stability that is measured by (6) for all four mentioned methods. In Figure 2, the stability as a function of the number of potential D2D transmitters around a receiver, the number of co-channel $\mathrm{D} 2 \mathrm{D}$ receivers, and the $\mathrm{D} 2 \mathrm{D}$ search radius 
are compared. In Figure 2a, the pairing stability in the FP method increases considerably when the number of potential transmitters around a receiver increases. The pairing stability increase of the MSP method is much less than the FP method, while in the CP and RP methods, this increase is negligible. In the FP and MSP methods, increasing the number of potential transmitters gives the receiver more proper choices.

In Figure 2b, the stability is illustrated for all four methods. The number of the D2D transmitters is 3 , and the number of receivers increase from 1 to 4 . Increasing the number of D2D receivers causes us to maintain the stability of the FP method, while it decreases sharply in the other three methods.

In Figure 2c, for a fixed number of D2D transmitters and receivers, the stability associated with increasing the search radius is calculated for all mentioned methods. Increasing the search radius does not have a significant effect on the stability of the FP, $\mathrm{CP}$, and RP methods; however, it reduces the stability of the MSP method. The stability of the MSP method decreases with increasing the $R_{\max }$ for two reasons. First, the users' data rates decrease with increasing $R_{\max }$, and second, the battery levels are not considered for this method.
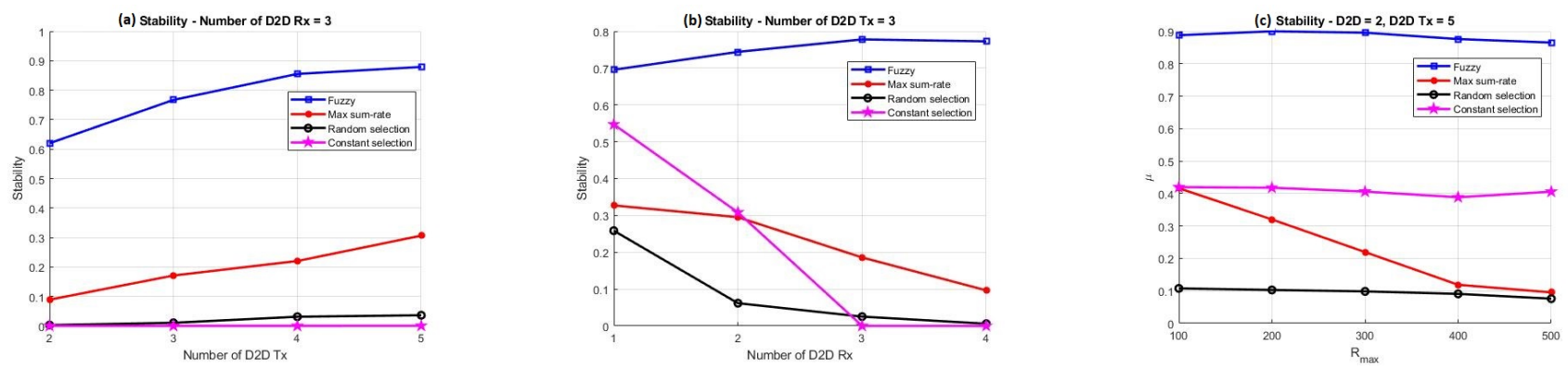

Figure 2. The stability as a function of (a) the number of potential D2D transmitters, (b) the number of co-channel D2D receivers, and (c) the D2D search radius.

In Figure 3, Jain's fairness index is computed for the number of potential D2D transmitters around a receiver, the number of co-channel D2D receivers, and the D2D search radius. We recall that Jain's fairness index can be computed as follows:

$$
\mathcal{J}\left(R_{1}, R_{2}, \ldots, R_{N}\right)=\frac{\left(\sum_{i=1}^{N} R_{i}\right)^{2}}{N \cdot \sum_{i=1}^{N} R_{i}^{2}}
$$

In Figure 3, it is illustrated that the FP method fairness index is maintained above all methods in a constant range, whether the number of receivers or transmitters or the D2D search radius increase. The fairness index in the MSP method behaves similarly to the FP method when $R_{\max }$ is constant. Nevertheless, increasing $R_{\max }$, the fairness index of the MSP method decreases sharply. By increasing $R_{\max }$, the range of changes in the data rate of co-channel users also increases and causes a reduction in the fairness index.

Increasing the number of transmitters has little effect on the fairness index of both $\mathrm{CP}$ and RP methods, while increasing the number of receivers reduces the fairness index of both methods. The reason can be found in the broader range of users' data rates. Furthermore, the fairness index of CP and RP methods reduces slightly with the increase of the D2D search radius. 

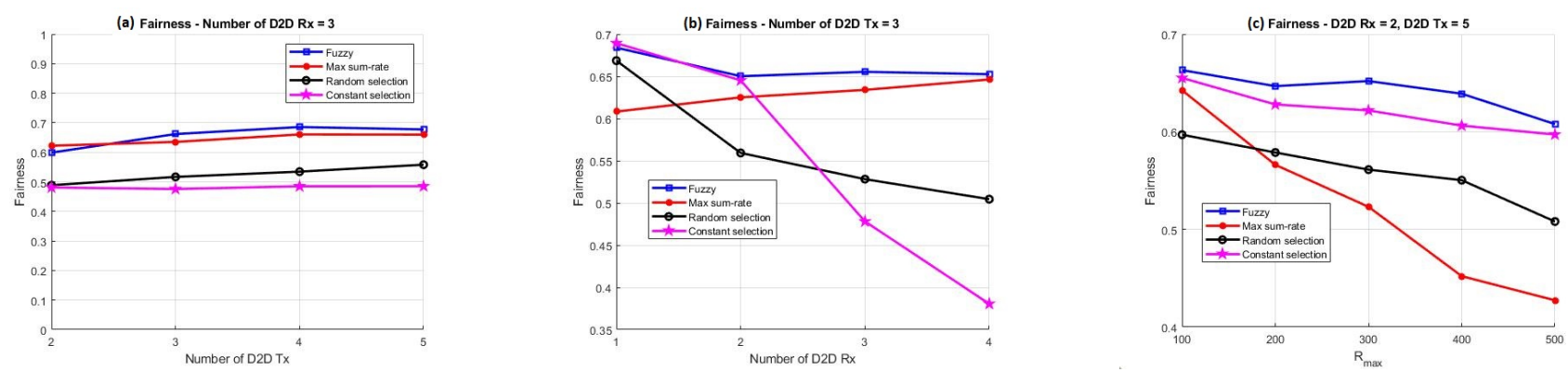

Figure 3. Jain's fairness is computed and compared according to (a) the number of potential D2D transmitters, (b) the number of co-channel D2D receivers, and (c) the D2D search radius.

Finally, in Figure 4, the sum rate as a function of the number of potential D2D transmitters around a receiver, the number of co-channel D2D receivers, and the D2D search radius are compared. In Figure 4a, the sum rates of all four methods are compared when the number of transmitters is increased. The MSP method has the highest data rate. Nevertheless, the FP method approaches the MSP method by increasing the number of potential transmitters. The other two methods (RP and CP) do not follow the MSP method in this case.

In Figure $4 b$, similar to Figure $4 a$, increasing the number of D2D receivers, the FP method sum rate follows the MSP method and also the sum rate of the RP method increases slowly, but after increasing the number of receivers (from 3 to 4), due to the effect of interference on the rate, the sum rate of the $\mathrm{CP}$ method tends to a constant value.

In Figure 4c, the sum rate is plotted as a function of the D2D search radius for the four methods mentioned. As expected, due to the increased interference, the sum rate decreases in all four methods as the search radius increases. However, the FP method is still the closest method to the MSP method.
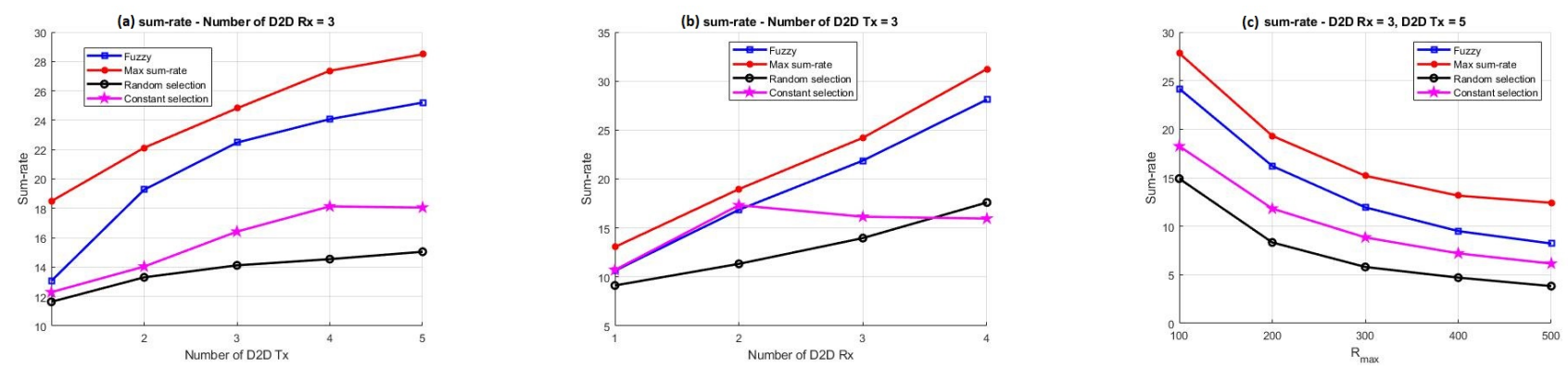

Figure 4. The sum rate as a function of (a) the number of potential D2D transmitters, (b) the number of co-channel D2D receivers, and (c) the D2D search radius.

\section{Applications}

The rapid spread of the disease has changed lifestyles and increased people's tendency to do things remotely. Under these conditions, the need to connect to communication networks has increased. With the advent of COVID-19, new technologies have tried to solve the crises behind it [41-44]. Among these technologies, 5G-related services also are useful in this manner. Such efforts can be seen in [45-48]. Given the new pandemic problems as well as the new technologies mentioned, more works are still needed to use the maximum capacity of these technologies in this regard. We shortly introduce one of the potential applications of D2D service to solve some pandemic problems in hospitals. The increase in the number of patients in hospitals has meant that hospital network resources generally do not meet the need for treatment during the pandemic. Therefore, services that can shift the focus away from cellular and centralized networks will be of particular importance. Suppose, for example, a hospital that receives a large number of patients but has limited network communication infrastructure to transfer patient data to physicians. 
D2D service can be one of the options to solve these challenges. Considering the two main goals of this paper, sustainable communication and the transmission of bulky contents, a scenario of joint resource allocation and pairing in a decentralized network based on D2D communications is presented in a typical hospital (Figure 5).

The hospital scenario shown in Figure 5 is in accordance with the proposed system model in Figure 1 with $N=2$ and $K=3$. In the mentioned hospital, physicians are considered as receivers ( $\mathrm{r} 1$ and $\mathrm{r} 2$ ) and hospital equipment is considered as transmitters $\left(t_{i j}\right)$. In this scenario, due to the large number of users in the hospital network, physicians use the cellular user resources instead of connecting to the network. They use the co-channel D2D links with the cellular user to connect to the nearby medical devices, considering the mentioned resource allocation and pairing protocols. Thus, the resource allocation and pairing mentioned in the system model can be implemented in a typical hospital. As a result, patient data transfer is done optimally and with an acceptable data rate.

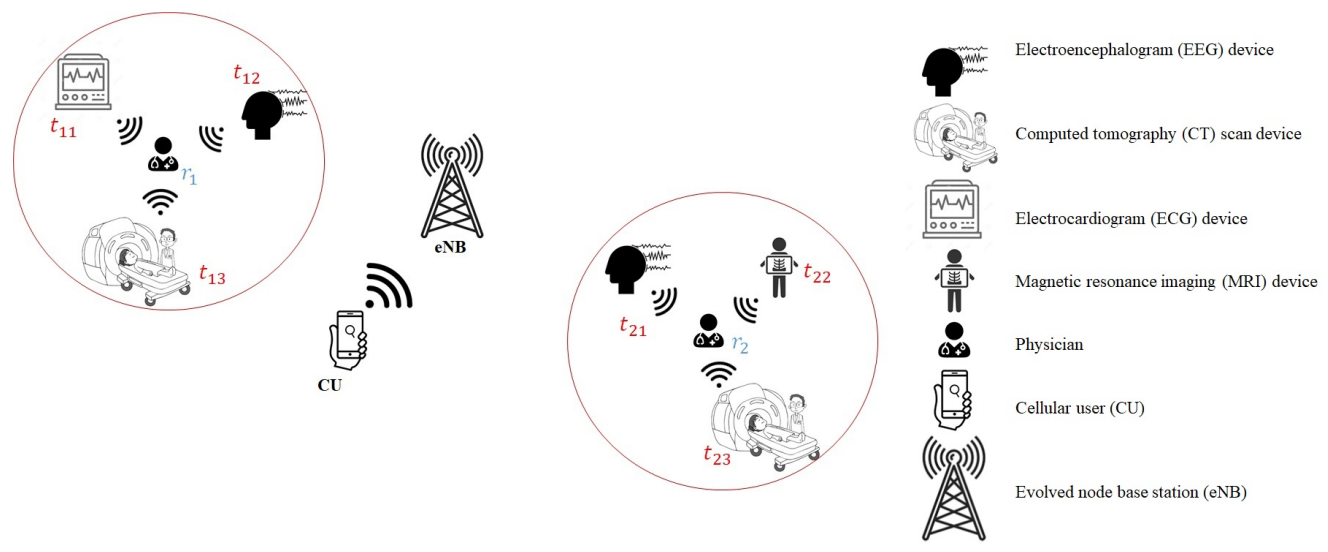

Figure 5. Hospital equipped with a decentralized network.

\section{Conclusions}

This study proposes a new method for joint multi-pairing and resource allocation in a single cell D2D scenario. Our method is based on fuzzy pairing criteria for selecting the best transmitter in a D2D receiver search radius. The set of transmitters is considered as a fuzzy set with respect to the data rates and battery levels of those transmitters. This fuzzy membership function can be seen as a stability index. The proposed method is compared with three other pairing methods: MSP, CP, and RP methods. The three parameters of stability, fairness, and sum rate in different modes, such as changing the D2D search radius and increasing the number of D2D transmitters and receivers for all four methods, are investigated. As a result, the fairness and stability of the fuzzy method work better than the other methods in different modes, and the FP method sum rate follows the MSP method sum rate.

Author Contributions: Investigation, A.P. and S.R.; Methodology, A.P., S.R., M.S. and M.F.; Supervision, A.P. and S.R.; Writing original draft, A.P. and S.R.; Writing review and editing, M.S. and M.F. All authors have read and agreed to the published version of the manuscript.

Funding: This research received no external funding.

Institutional Review Board Statement: Not applicable.

Informed Consent Statement: Not applicable.

Data Availability Statement: The study did not report any data.

Conflicts of Interest: The authors declare no conflict of interest. 


\section{References}

1. Mach, P.; Becvar, Z.; Vanek, T. In-band device-to-device communication in OFDMA cellular networks: A survey and challenges. IEEE Commun. Surv. Tutor. 2015, 17, 1885-1922. [CrossRef]

2. Jameel, F.; Hamid, Z.; Jabeen, F.; Zeadally, S.; Javed, M.A. A survey of device-to-device communications: Research issues and challenges. IEEE Commun. Surv. Tutor. 2018, 20, 2133-2168. [CrossRef]

3. Wei, G.; Zhao, X. Induced hesitant interval-valued fuzzy Einstein aggregation operators and their application to multiple attribute decision making. J. Intell. Fuzzy Syst. 2013, 24, 789-803. [CrossRef]

4. Wang, L.; Wu, H. Fast pairing of device-to-device link underlay for spectrum sharing with cellular users. IEEE Commun. Lett. 2014, 18, 1803-1806. [CrossRef]

5. Gu, Y.; Zhang, Y.; Pan, M.; Han, Z. Cheating in matching of device to device pairs in cellular networks. In Proceedings of the 2014 IEEE Global Communications Conference, Austin, TX, USA, 8-12 December 2014.

6. Wang, L.; Stuber, G.L. Pairing for resource sharing in cellular device-to-device underlays. IEEE Netw. 2016, 30, 122-128. [CrossRef]

7. Li, Y.; Song, C.; Jin, D.; Chen, S. A dynamic graph optimization framework for multihop device-to-device communication underlaying cellular networks. IEEE Wirel. Commun. 2014, 21, 52-61. [CrossRef]

8. Choi, K.W.; Han, Z. Device-to-device discovery for proximity-based service in LTE-advanced system. IEEE J. Sel. Areas Commun. 2014, 33, 55-66. [CrossRef]

9. Xu, C.; Gao, C.; Zhou, Z.; Chang, Z.; Jia, Y. Social network-based content delivery in device-to-device underlay cellular networks using matching theory. IEEE Access 2016, 5, 924-937. [CrossRef]

10. Miao, L.; Bai, B.; Chen, W. 4-DMWM approach for caching based optimal D2D pairing and channel allocation: Centralized and distributed algorithm design. IEEE Access 2016, 4, 9213-9224. [CrossRef]

11. Pourmoslemi, A.; Rajabi, S.; Salimi, M. Selecting the Best Transmitter in Wireless Device-to-Device Communications Using a Fuzzy Decision-Making Method. In Proceedings of the International Online Conference on Intelligent Decision Science, Istanbul, Turkey, 7-8 August 2020; Springer: Berlin/Heidelberg, Germany.

12. Rajabi, S.; Ghorashi, S.A.; Shah-Mansouri, V. Impact of connecting to the nth nearest node in dedicated device-to-device communications. Electron. Lett. 2018, 54, 535-537. [CrossRef]

13. Rashed, S.K.; Asvadi, R.; Rajabi, S.; Ghorashi, S.A.; Martini, M.G. Power allocation for D2D communications using max-min message-passing algorithm. IEEE Trans. Veh. Technol. 2020, 69, 8443-8458. [CrossRef]

14. Zhou, Z.; Kaoru, O.; Dong, M.; Chen, X. Energy-efficient matching for resource allocation in D2D enabled cellular networks. IEEE Trans. Veh. Technol. 2020, 66, 5256-5268. [CrossRef]

15. Yuan, Y.; Yang, T.; Hu, Y.; Feng, H.; Hu, B. Two-Timescale Resource Allocation for Cooperative D2D Communication: A Matching Game Approach. IEEE Trans. Veh. Technol. 2020, 70, 543-557. [CrossRef]

16. Zhou, Z.; Ma, G.; Dong, M.; Ota, K.; Xu, C.; Jia, Y. Iterative energy-efficient stable matching approach for context-aware resource allocation in D2D communications. IEEE Access 2016, 4, 6181-6196. [CrossRef]

17. Ma, R.; Chang, Y.J.; Chen, H.H.; Chiu, C.Y. On relay selection schemes for relay-assisted D2D communications in LTE-A systems IEEE Trans. Veh. Technol. 2017, 66, 8303-8314. [CrossRef]

18. Gandotra, P.; Jha, R.K. Zonal-based GrEEn algorithm for augmenting the battery life in spectrum shared networks via D2D communication. IEEE Trans. Veh. Technol. 2018, 68, 405-419. [CrossRef]

19. Chaudhari, A.; Gandikota, J.; Sen, A.; Narayan, S. A Realistic Approach to Enhance the Battery Performance of Device-to-Device (D2D) Relay UEs. In Proceedings of the 17th Annual Consumer Communications \& Networking Conference (CCNC), Las Vegas, NV, USA, 10-13 January 2020.

20. Zadeh, L.A. Fuzzy sets. Inf. Control. 1965, 8, 338-353. [CrossRef]

21. Cho, Y.J.; Rassias, T.M.; Saadati, R. Fuzzy Operator Theory in Mathematical Analysis; Springer: Berlin/Heidelberg, Germany, 2018.

22. Bellman, R.; Kalaba, R.; Zadeh, L. Abstraction and pattern classification. J. Math. Anal. Appl. 1966, 13, 1-7. [CrossRef]

23. Bellman, R.E.; Zadeh, L.A. Decision-making in a fuzzy environment. Manag. Sci. 1970, 17, B-141. [CrossRef]

24. Zadeh, L.A. Fuzzy sets and their application to pattern classification and clustering analysis. In Classification and Clustering; Elsevier: Amsterdam, The Netherlands, 1977; pp. 251-299.

25. Bezdek, J.C. Fuzzy Mathematics in Pattern Classification. Ph.D. Thesis, Applied Mathematics, Cornell University, Ithaca, NY, USA, 1973.

26. Dunn, J.C. A fuzzy relative of the ISODATA process and its use in detecting compact well-separated clusters. J. Cybern. 1973, 3, 32-57. [CrossRef]

27. Mamdani, E.H.; Assilian, S. An experiment in linguistic synthesis with a fuzzy logic controller. Int. J. Man-Mach. Stud. 1975, 7, 1-13. [CrossRef]

28. Kosko, B. Neural Networks and Fuzzy Systems: A Dynamical Systems Approach to Machine Intelligence; Prentice Hall: Hoboken, NJ, USA, 1992

29. Ahvar, E.; Pourmoslemi, A.; Piran, M.J. FEAR: A fuzzy-based energy-aware routing protocol for wireless sensor networks. Int. J. Grid Comput. Appl. 2011, 7. [CrossRef]

30. Bělohlávek, R.; Dauben, J.W.; Klir, G.J. Fuzzy Logic and Mathematics: A Historical Perspective; Oxford University Press: Oxford, UK, 2017. 
31. Wei, W.; Li, Z.; Li, Z.; Sha, X. Fuzzy logic power control of device to device communication underlay td-lte-a system. In Proceedings of the 3rd International Conference on Consumer Electronics, Communications and Networks, Xianning, China, 20-22 November 2013.

32. Li, H.; Chen, X.; Zhang, Y.; Wang, P.; Qiang, W.; Liu, N. Fuzzy mathematics and game theory based D2D multicast network construction. J. Syst. Eng. Electron. 2019, 30, 13-21.

33. Liu, M.; Liu, Y.; Zhou, Y.; Zhang, W.; Yu, Y. Research on D2D resource allocation algorithm based on improved fuzzy clustering. In Proceedings of the Tenth International Conference on Digital Image Processing (ICDIP 2018), Shanghai, China, 11-14 May 2018.

34. Chen, P.; Xue, J.; Chen, Y.; Wei, S.; Ji, Y. Resource allocation algorithm based on Fuzzy cluster grouping for device-to-device communication. In Bio-Inspired Computing-Theories and Applications; Springer: Berlin/Heidelberg, Germany, 2015.

35. Subramani, M.; Kumaravelu, V.B. A quality-aware fuzzy-logic-based vertical handover decision algorithm for device-to-device communication. Arab. J. Sci. Eng. 2019, 44, 2413-2425. [CrossRef]

36. Klement, E.P.; Mesiar, R.; Pap, E. Triangular Morms; Springer Science \& Business Media: Berlin/Heidelberg, Germany, 2013.

37. Schweizer, B. Associative funcrions and statistical triangle inequalities. Puble. Math. Debr. 1961, 8, 169-186.

38. Munir, M.; Kalsoom, H.; Ullah, K.; Mahmood, T.; Chu, Y.-M. T-spherical fuzzy Einstein hybrid aggregation operators and their applications in multi-attribute decision making problems. Symmetry 2020, 12, 365. [CrossRef]

39. Zhao, X.; Wei, G. Some intuitionistic fuzzy Einstein hybrid aggregation operators and their application to multiple attribute decision making. Knowl.-Based Syst. 2013, 37, 472-479. [CrossRef]

40. Lin, X.; Andrews, J.G.; Ghosh, A. Spectrum sharing for device-to-device communication in cellular networks. IEEE Trans. Wirel. Commun. 2014, 13, 6727-6740. [CrossRef]

41. Muhammad, G.; Alqahtani, S.; Alelaiwi, A. Pandemic Management for Diseases Similar to COVID-19 Using Deep Learning and 5G Communications. IEEE Netw. 2021, 35, 21-26. [CrossRef]

42. Siriwardhana, Y.; De Alwis, C.; Gür, G.; Ylianttila, M.; Liyanage, M. The fight against the COVID-19 pandemic with 5G technologies. IEEE Eng. Manag. Rev. 2020, 48, 72-84. [CrossRef]

43. Patzold, M. The Role of 5G in Limiting the Impact of the COVID-19 Pandemic [Mobile Radio]. IEEE Veh. Technol. Mag. 2020, 15, 6-12. [CrossRef]

44. Ye, B.; Yuan, X.; Cai, Z.; Lan, T. Severity Assessment of COVID-19 Based on Feature Extraction and V-Descriptors. IEEE Trans. Ind. Inform. 2021, 17, 7456-7467. [CrossRef]

45. Lin, J.C. Telecommunications health and safety: The Covid-19 pandemic and 5G cellular telecommunication systems. URSI Radio Sci. Bull. 2020, 372, 56-59. [CrossRef]

46. Ren, H.; Shen, J.; Tang, X.; Feng, T. 5G Healthcare Applications In COVID-19 Prevention And Control. In Proceedings of the 2020 ITU Kaleidoscope: Industry-Driven Digital Transformation (ITU K), Ha Noi, Vietnam, 7-11 December 2020; pp. 1-4.

47. Wang, P.; Zhao, Y. Research on the Development of 5G under the Background of the COVID-19 Epidemic. In Proceedings of the 2021 2nd International Conference on E-Commerce and Internet Technology (ECIT), Hangzhou, China, 5-7 March 2021; pp. 34-137.

48. Chamola, V.; Hassija, V.; Gupta, V.; Guizani, M. A comprehensive review of the COVID-19 pandemic and the role of IoT, drones, $\mathrm{AI}$, blockchain, and 5G in managing its impact. IEEE Access 2020, 8, 90225-90265. [CrossRef] 